\title{
Doppler Lidar System Design via Interdisciplinary Design Concept at NASA Langley Research Center - Part I
}

\author{
Charles M. Boyer ${ }^{1}$, Trevor P. Jackson ${ }^{2}$, Jeffrey Y. Beyon ${ }^{3}$, and Larry B. Petway ${ }^{4}$ \\ ${ }^{1}$ NASA Langley Research Center, MS 432, Hampton, VA 23681, USA, Charles.M.Boyer@nasa.gov \\ ${ }^{2}$ Mechanical Engineering Department, Old Dominion Univ., Norfolk, VA 23529, USA, tjack040@odu.edu \\ ${ }^{3}$ NASA Langley Research Center, MS 488, Hampton, VA 23681, USA, Jeffrey.Y.Beyon@nasa.gov \\ ${ }^{4}$ NASA Langley Research Center, MS 468, Hampton, VA 23681, USA, Larry.B.Petway@nasa.gov
}

\begin{abstract}
Optimized designs of the Navigation Doppler Lidar (NDL) instrument for Autonomous Landing Hazard Avoidance Technology (ALHAT) were accomplished via Interdisciplinary Design Concept (IDEC) at NASA Langley Research Center during the summer of 2013. Three branches in the Engineering Directorate and three students were involved in this joint task through the NASA Langley Aerospace Research Summer Scholars (LARSS) Program. The Laser Remote Sensing Branch (LRSB), Mechanical Systems Branch (MSB), and Structural and Thermal Systems Branch (STSB) were engaged to achieve optimal designs through iterative and interactive collaborative design processes. A preliminary design iteration was able to reduce the power consumption, mass, and footprint by removing redundant components and replacing inefficient components with more efficient ones. A second design iteration reduced volume and mass by replacing bulky components with excessive performance with smaller components custom-designed for the power system. Mechanical placement collaboration reduced potential electromagnetic interference (EMI). Through application of newly selected electrical components and thermal analysis data, a total electronic chassis redesign was accomplished. Use of an innovative forced convection tunnel heat sink was employed to meet and exceed project requirements for cooling, mass reduction, and volume reduction. Functionality was a key concern to make efficient use of airflow, and accessibility was also imperative to allow for servicing of chassis internals. The collaborative process provided for accelerated design maturation with substantiated function.
\end{abstract}

Keywords: ALHAT, Doppler Lidar, IDEC, Langley, LARSS

\section{INTRODUCTION}

Three branches of different disciplines in the Engineering Directorate (ED) at NASA Langley Research Center (LaRC) teamed up during the summer of 2013 to analyze and optimize an existing electronics chassis through redesign. The collaborative efforts were based on the Interdisciplinary Design Concept (IDEC) which focuses on maximizing the productivity by combining multiple disciplines and their best engineering practices. The goal of the team was to review and improve the design of the NDL instrument [1] for ALHAT. The chassis was redesigned to ensure cooling by initially overestimating heat sink and fan requirements. Another goal was to reduce excess capability to maximize the efficiency of the chassis in power consumption, mass, and volume while considering other ways to optimize functionality. The electronics chassis is currently designed to work on the Morpheus Test Bed, thus the scope of the project was concentrated on operation in atmospheric conditions. Morpheus is a rocket-powered terrestrial free-flyer vehicle built by NASA Johnson Space Center (JSC). The ALHAT Lidar system has years of testing and maturation [2] to progress towards its final, space-ready form. Having an efficient system for ground testing will prove to be very valuable. Through analysis and optimization of the current system, the outcome of this effort will make the transition to a space-qualified system more comprehensive. Initial project requirements were a reduction in power consumption of $20 \%$ from an initial power usage, a reduction in mass of $15 \%$ from an initial mass, and a reduction in volume of $20 \%$ from an initial volume. The system must be capable of operation between $-40{ }^{\circ} \mathrm{C}$ and $50{ }^{\circ} \mathrm{C}$.

This paper consists of three parts: Parts I - III. Each part is presented in a separate document. Part I presents the introduction of IDEC and the mechanical design aspect of this task. Part II discusses the thermal design aspect, and Part III focuses on the electrical design aspect. The final design outcome and comparison are presented in Part III. 


\section{INTERDISCIPLINARY DESIGN CONCEPT (IDEC)}

Figure 1 illustrates the IDEC. Design requirements are established based on science, engineering, and business requirements. Once the design requirements are identified, a team is formed, consisting of members from different disciplines. They collaborate to achieve a final design through an iterative process. Each discipline has a small group of engineers and a lead who organizes and represents the design activities within the discipline. At the design requirement level, there is a project manager who approves the outcome of each design iteration and the escalation to the next design level. The uniqueness of the IDEC is the iterative feedback process being embedded in each level of design, which allows fine-tuning of the process in early stage of design steps. This will help reduce design costs and increase the level of understanding the design specification for each and all team members.

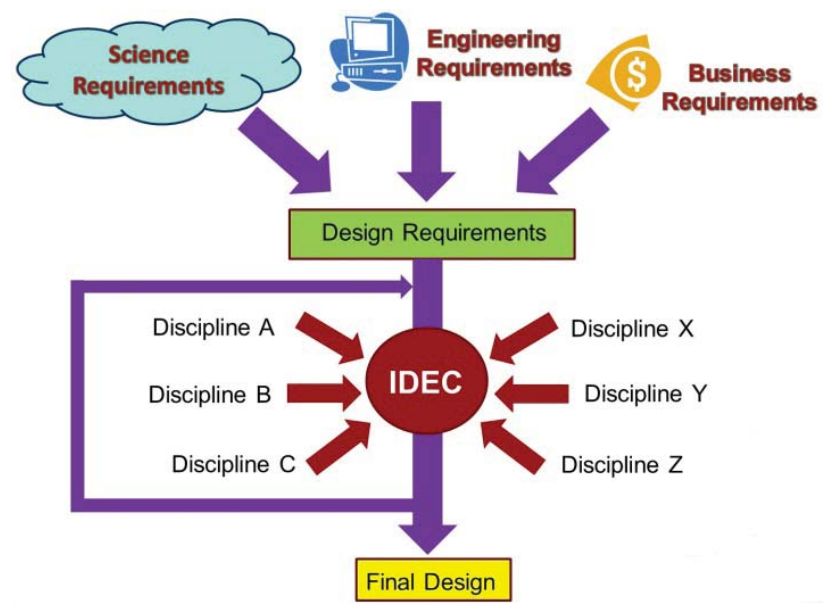

Figure 1 Interdisciplinary Design Concept (IDEC)

In the summer of 2013, an IDEC team from three disciplines such as electrical, thermal, and mechanical areas was formed to apply the IDEC design principle to improve the NDL instrument for ALHAT. Each discipline had a LaRC civil servant and a university student from the LaRC's LARSS program. The role of LaRC in ALHAT is to develop three Lidar sensors, which are a 3-D imaging Flash Lidar sensor, a Doppler Lidar sensor, and a Laser Altimeter. The three sensors will perform five essential landing functions such as Altimetry, Velocimetry, Terrain Relative Navigation (TRN), Hazard Detection and Avoidance (HAD), and Hazard Relative Navigation (HRN) [3]. The NDL instrument is part of the LaRC's ALHAT instrument and the IDEC team's goal was to reduce mass, volume, and power of NDL instrument by improving its design through a collaborative iterative process.

\section{MECHANICAL ASPECTS OF DESIGN}

Documentation was not readily available for the existing chassis. Before conducting a redesign, work was done to understand the chassis thoroughly. Organization of this information was crucial in disseminating system specifications to design team members. This was completed through identification of part numbers from the physical chassis, documentation that was available, and inquiry with employees familiar with the chassis to attain part numbers not readily available. These part numbers were then researched through use of data sheets or contact with manufacturers. Information about part numbers, descriptions, mass, size dimensions, maximum operating temperatures, and power dissipation for each component was collected and compiled through collaboration across the disciplines. This collection of information helped make the redesigns of the chassis more focused and efficient.

There are currently two electronics chassis that perform the same function. The first one built, referred to as "Doppler 1", is in use at Johnson Space Center in Houston, TX [4] and is the chassis that is currently run on the Morpheus Test Bed. The second chassis, which is referred to as "Doppler 2" or "original," is currently located at Langley Research Center (LaRC) in Hampton, VA. The two models are not identical. The second chassis located at LaRC is more up-to- 
date. A “.step” format CAD model of Doppler 1 was provided to help with the analysis of the Doppler 2 chassis. The model, however, was not a complete representation. Some components differ between Doppler 1 and Doppler 2. Pro-E was employed to modify the Doppler 1 model into a useable model for Doppler 2.

The initial Doppler 1 CAD model was analyzed to ensure accuracy. The “.step” file contains no information other than length dimensions and does not have proper datum relations. This means that modification of the model assembly will cause failure. Comparison measurements were taken on the physical Doppler 2 chassis and compared to measurements taken from the Doppler 1 CAD model to ensure the accuracy of the model. Once these were verified, the individual components that existed in both the Doppler 1 and the Doppler 2 chassis were assigned proper datum relations. The individual components were also assigned with their part numbers, part descriptions, and masses obtained during the research of the physical chassis directly in the model. The parts that did not exist in the Doppler 1 CAD model or needed modification were then produced and also assigned proper datum relations. The Doppler 2 CAD model was then created through total reassembly of the chassis from scratch. This ensured proper datum relations throughout the entire model. The Doppler 2 CAD model then provided a comprehensive overview of the Doppler 2 physical chassis, while also being readily modifiable.

The information obtained and organized in producing the Doppler 2 CAD model was crucial to the redesign of the chassis. Understanding the existing chassis in detail allowed future redesigns to retain this level of detail for a more realistic redesigned model. Component identification and placement helped the electrical and thermal analysis greatly. The Doppler 2 CAD model helped obtain the volume of individual components, as well as the overall chassis. The Doppler 2 CAD model also sought to attain a realistic representation of the mass. The mass was calculated through a combination of using assigned density properties for components that were a solid material and assigned masses for electrical components that had a variety of materials. The masses for the electrical components were taken from data sheets or from a contact with manufacturers. The Doppler 2 CAD model achieved a mass of $44.6 \mathrm{lbs}$, with an estimation on the wiring and connectors of $2.2 \mathrm{lbs}$. The Doppler 2 physical chassis was then weighed in-house at $45.2 \mathrm{lbs}$. With the estimation of the wiring and connectors included, the difference between the Doppler 2 CAD model and the physical Doppler 2 is less than $5 \%$.

The Doppler 2 CAD model with component identification was used for a baseline thermal analysis. It provided component identification, placement, material, and power dissipation for input into a program called Thermal Desktop. The baseline thermal test showed that the chassis was not exceeding maximum operating temperatures and was actually "overkill". The redesign process then focused on how to remove extraneous mass, volume, and power consumption while still having a system that functions at specification.

\section{DESIGN ANALYSIS CYCLE (DAC) 1}

For Design Analysis Cycle (DAC) 1, the model was thermally driven. Maximizing fan airflow over the heat sink surface area was important for the reduction of volume, mass, and power. Use of a tunnel shaped heat sink with components attached to the exterior surface and a fan located at each end to "push" and "pull" air through proved to be most effective (see Figure 2). A thermal interface material, which is currently used on the existing hardware, is placed between the components and the heat sink to improve heat transfer. Other parts that did not produce heat were not mounted to the heat sink. Some of these other parts include fiber optic cables, wiring and connectors, a plastic fiber wrap, and an aluminum casing to protect the internals of the system. The tunnel design creates forced convection through the tunnel, which utilizes much more of the fan airflow than the existing physical chassis. Because of this four of the six fans could be removed. The components conduct heat directly into the heat sink. The heat sink then dissipates the heat utilizing airflow from the fans. The tunnel has vertical fins, which increase the convective surface area. The advantage of all the components sharing a single heat sink is that the overall thermal capacity of the heat sink is much higher than if each component or groups of components had their own heat sink. The Doppler 2 chassis has a unified heat sink, however, and the DAC 1 design utilizes not only a unified heat sink, but the tunnel shape to use the fan airflow much more efficiently than the current design.

The containment of the heat sink within the chassis not only reduces volume, but it also improves heat sink efficiency. Morpheus tests are run in Houston, Texas during the summer months. An aluminum heat sink exposed to the sun for 
extended periods during the tests is not conducive to an efficient cooling system. During the Morpheus tests there is also a large amount of heat generated by the engine. Having the heat sink contained within the chassis helps to shield it from these thermal factors in the surrounding environment, allowing the heat sink to function more effectively.

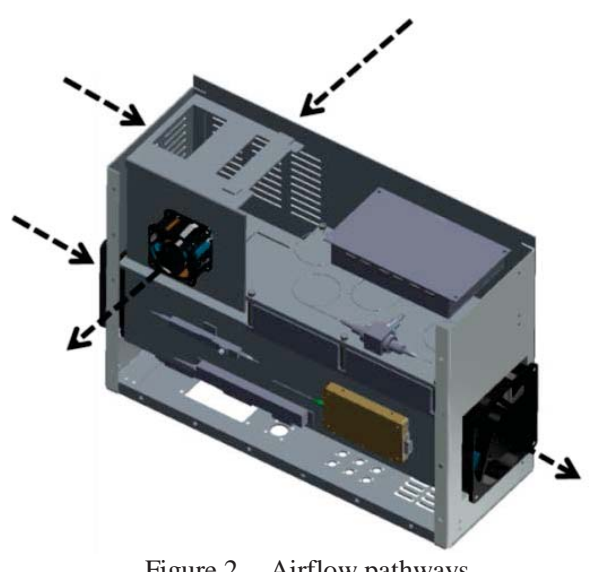

Figure 2 Airflow pathways

For DAC 1 endeavors were made to retain the bottom face of the case in an effort to not only save cost, but also make the mounting configuration and hardware remain the same for attachment to the Morpheus Test Bed. The volume was still reduced due to the fact that the heat sink and fans were no longer a protrusion on the exterior of the case. The case also achieved a slightly shorter stature. Although initial attempts were made to retain the walls of the case and modify them to fit the fans at each end of the heat sink tunnel, this proved to be restrictive to the design size and a new case was necessary. Organization of the internal components was important. In the existing chassis, the Command and Data Handling Unit (C\&DH) has its own independent fan. The path for the airflow of this fan was inefficient, with no clear inlet or outlet for the flow. DAC 1 sought to correct this with inlet vents in the case and a direct outlet from the fan as shown in Figure 2. This averted extraneous heat being held in the interior of the chassis during operation. The C\&DH unit also has a conductive heat sink on its bottom face which will be mated directly to the heat sink tunnel, providing multiple forms of cooling for the C\&DH unit.

During the Morpheus tests, airborne debris are present due to lift off and landing. The inlet vents and opening for exit flow from the C\&DH expose the interior of the chassis to these debris. To remedy this, a filter system was implemented on the C\&DH fan as shown in Figure 3. The filter system is produced by EBM Papst [6], who also produces the current fan. This means the cost of implementation for the fan filter system is extremely low. The filter system has a changeable filter medium material, which is extremely affordable and could be replaced after each test. Due to the large amount of debris in the air during the Morpheus tests, analysis was conducted to ensure that the filter could operate properly throughout the entirety of the tests without becoming clogged, inhibiting airflow as a result. Different filter medium material types can also be used to fine tune the trade-offs between particulate filtration ability and airflow reduction. Filtration was also necessary for the inlet vents on the case. Figure 4 shows that a frame was placed around the vents in which a rectangular piece of filter medium would be slid into and secured with retainers at the top. Due to the pliable nature of the filter medium, the frame into which the filter medium is placed will have a tight fit to ensure a good seal. This prevents debris from going around the filter medium and into the interior of the chassis during operation.

Effort was also made to keep the interior of the chassis clear of debris through the modification of the case inlet for the fans and tunnel heat sink. The inlet shape was designed to be circular as shown in Figure 3 to let in the maximum amount of airflow while maintaining a good seal from the fans to the case. The heat sink tunnel system is designed to be a closed system, meaning all air that goes in the inlet goes through the outlet with no exposure to the internal components of the chassis. Because of this, filtration on the heat sink tunnel fans was not employed. The reduction in airflow through the heat sink tunnel due to filtration was large enough to dissuade the use of a filter system for the heat sink fans. The fans can be disassembled from the heat sink tunnel to provide cleaning of the interior heat sink fins if eventual particle build up becomes an issue. All fans were made to be flush with the case, not only to have a good seal around the edges of the fan to ensure no foreign debris entered around the fan, but also to make sealing of all orifices on 
the chassis easy for shipping purposes.

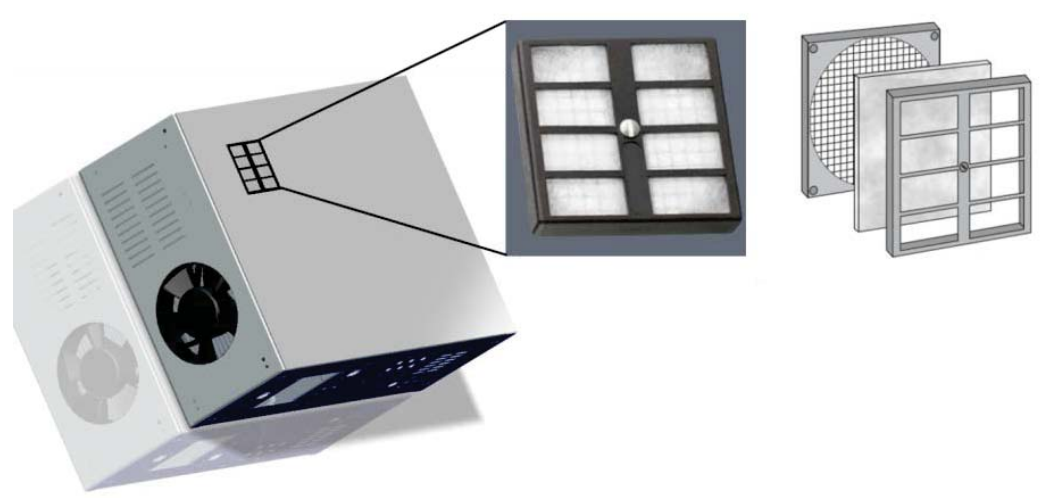

Figure 3 Filtration system on C\&DH fan

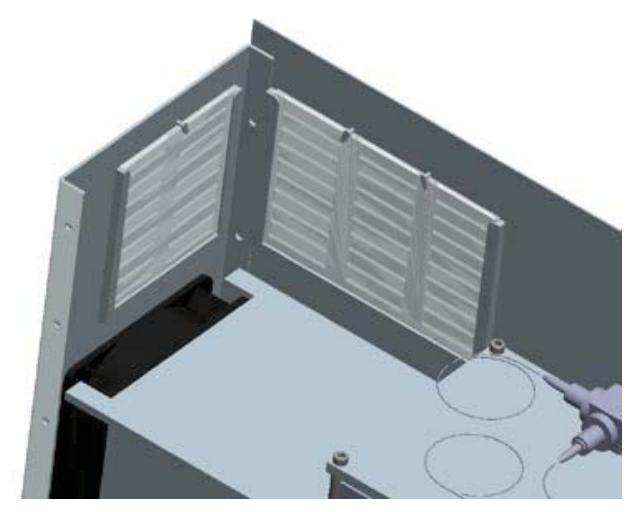

Figure 4 Filtration system on C\&DH inlets

Thermal testing was done on the DAC 1 heat sink tunnel to verify that the design would work. The DAC 1 heat sink tunnel proved to be more than capable at rejecting the heat generated by the system. There was also modification of the electrical system to remove redundant components, which was reflected in the DAC 1 CAD model. Through the aforementioned modifications, a reduction in power of $37 \%$, a reduction in mass of $22 \%$, and a reduction in volume of $22 \%$ were achieved. These reductions met and exceeded design requirements. The power reduction was mainly attributed to the removal of four fans. The mass and volume reduction was attributed to the maximized efficiency of the heat sink shape and size.

\section{DESIGN ANALYSIS CYCLE (DAC) 2}

DAC 2 sought even more aggressive reductions. A cube shape was used for further reduction in volume. Through electrical component removal and replacement by smaller footprint parts, all components could now fit on a shorter heat sink. Shortening of the heat sink provided for better airflow through the heat sink, allowing reduction to one fan for the heat sink tunnel. Through these modifications a reduction from the baseline model of $45 \%$ in power, $29 \%$ in mass, and $26 \%$ in volume was achieved.

DAC 2 retained an airflow inlet and an outlet for the C\&DH system, as well as the filtration system and a fan to case fitment. The cube-shaped case provided better internal component fitment with room to spare. The additional room in the chassis will accommodate the organization of the optical fiber. DAC 2 component placement to the external surface of the heat sink tunnel was reorganized to ensure optimized thermal and electrical functionality. Components were placed close to the hardware they supported to reduce wiring length for electromagnetic interference (EMI) considerations. For thermal optimization components were placed according to heat dissipation, with hotter components segregated from the rest to help share the thermal capacity of the entire heat sink more evenly. 
Disassembly of the chassis for maintenance was also considered. The case consists of six parts. All parts can be unbolted and removed individually for access to specific components directly. Because the heat sink tunnel has components attached to all four sides it was very important to have the ability to remove individual case parts while the system retains rigidity. The tunnel heat sink is made of aluminum and bolted directly to the case with the fan inset. This allows for the tunnel heat sink to have a stable attachment to the case and provides the core strength from a structural standpoint. The cubic shape of DAC 2 creates a more stable configuration with a lower center of gravity than a tall slim design, which was used in the original baseline and DAC 1 . The chassis is mounted to Morpheus by vibration isolators so that vibration is a less prominent concern.

Post DAC 2 research sought to fine tune the system and continue improvements. Modification of the fan type could find a balance between power consumption and amount of airflow. A variety of fan types and manufacturers were reviewed. Although the current fans on the physical chassis met airflow requirements for proper function of the heat sink tunnel, higher flowing fans could also be utilized. Inversely, lower airflow fans could be utilized to reduce power, although the power reduction by reducing five of the six fans proved to be much more significant than the reduction in power of the single remaining fan. Because the current fans have already been purchased, a decision was made to continue throughout the design process with the same fan model. The fact that the five removed fans can be used as backup fans was also a driving factor in retaining the same fan model.

There are extruded aluminum heat sink tunnels produced specifically for direct fan attachment available from manufacturers which resemble the DAC 1 and DAC 2 heat sink tunnels. These tunnels have a variety of fin shapes and configurations, however, and the manufactured tunnels would need significant modification to match the functionality of the DAC 1 and DAC 2 heat sinks, resulting in additional mass and lower thermal efficiency at attachments.
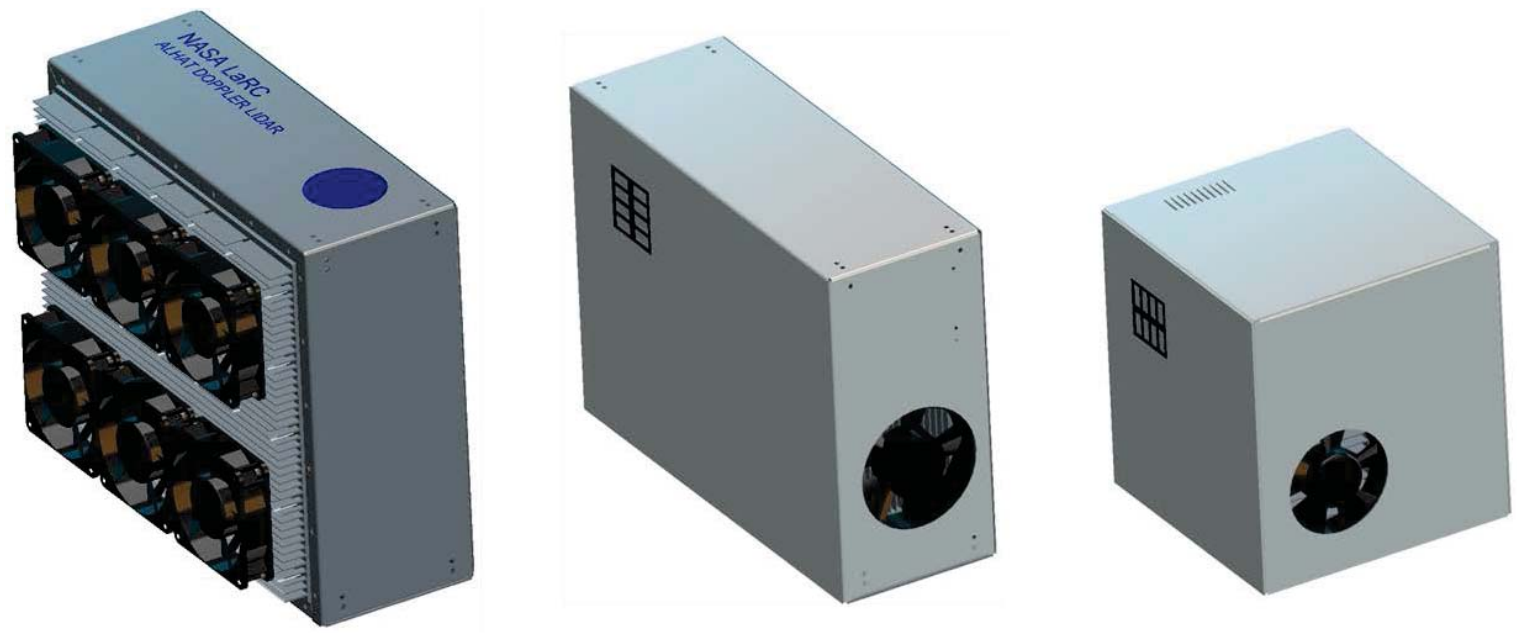

Figure 5 From left to right, original, DAC 1, and DAC 2 designs. The approximate volumes of each are 2,200 cubic inch (original), 1,700 cubic inch (DAC 1), and 1,600 cubic inch (DAC 2).

\section{CONCLUSION}

A multitude of possible modifications to the chassis in the future exists. Further analysis could continue reductions in mass and volume. The greatest contributor to reductions in the future will be component replacements or reconfigurations, most notably the C\&DH unit and all fiber optic equipment in the chassis. These systems were completely untouched during analysis. Modification or replacement of components in these two systems was not part of the design requirement and placed outside the scope of the project. There has been discussion of reductions in the fiber optic system, especially in regard to the beam splitters and fiber wrap as well as the connector rack. Reduction in these two components as well as reduction or reconfiguration of the C\&DH unit could provide for a much smaller and lighter chassis redesign. With these components reduced, a tunnel size reduction and subsequent use of a smaller fan could 
provide for further reductions in mass, volume, and power. Different materials for the case could also be utilized. A combination of an aluminum skeleton with plastic paneling or other materials could be used. Due to the flammable nature of the Morpheus this design was not pursued although further research could prove it to be viable.

\section{ACKNOWLEDGMENTS}

The authors are grateful for a great deal of guidance and encouragement from the NASA LaRC ALHAT Principal Investigator Dr. Farzin Amzajerdian and the branch heads Ms. Jill Prince, Mr. Steven Gayle, and Dr. Larry Petway in the NASA LaRC Engineering Directorate.

\section{BIBLIOGRAPHY}

[1] Farzin Amzajerdian, Diego Pierrottet, Larry Petway, Glenn Hines, and Bruce Barnes, "Doppler lidar sensor for precision navigation in GPS-deprived environment,” Proc. of SPIE ,Vol. 8731, (2013).

[2] Diego Pierrottet, Farzin Amzajerdian, Larry Petway, Bruce Barnes, George Lockard, and Glenn Hines, "Navigation Doppler Lidar Sensor for Precision Altitude and Vector Velocity Measurements Flight Test Results," Proceeding SPIE, Vol. 8044, (2011).

[3] Farzin Amzajerdian, Diego Pierrottet, Larry Petway, Glenn Hines, Vincent Roback and Robert Reisse, "Lidar Sensors for Autonomous Landing and Hazard Avoidance,” AIAA Space Conference Proceedings, (2013).

[4] Rutishauser, D., Epp, C. D., and Robertson, E. A., "Free-Flight Terrestrial Rocket Lander Demonstration for NASA's Autonomous Landing and Hazard Avoidance Technology (ALHAT) System,” Proc. of AIAA SPACE, 2012.

[5] 3M Company, “3M"M Thermally Conductive Silicone Interface Pads.” St. Paula, MN August 2009.

[6] “Compact fans for AC and DC.” ebm-papst. Web. 2011. < http://www.ebmpapst.se/sv/dat/media_manager /news/145/news-files/Compactfans2011.pdf>.

[7] Çengel, Y. A., Ghajar, A. J. (2011). Heat and Mass Transfer: Fundamentals and applications (4 ${ }^{\text {th }}$ ed.). New York, NY: The McGraw-Hill Companies, Inc.

[8] http://www.srh.noaa.gov/hgx/?n=climate_iah_normals_aug

[9] Czarkowski, Dariusz , and Marian K. Kazimierczuk. Resonant Power Converters. 2nd. Hoboken, New Jersey: John Wiley \& Sons, 2011. Page 487.

[10] Trzynadlowski, Andrzej M. "EMI Effects of Power Converters." In Power Electronics Handbook, edited by Muhammad H. Rashid, 1116. Boston: Academic Press, 2007. Page 1116. 